\title{
REPORT OF A REVIEW OF THE NSF OCEAN SCIENCES RESEARCH SECTION
}

\author{
By Robert Duce, Sallie Chisholm, Thomas Kinder, Peter Liss, Ted Moore and Mary Scranton
}

\section{E} VERY THREE YEARS a Committee of Visitors is formed by the National Science Foundation (NSF) Advisory Committee on Geosciences to review the various aspects of NSF funding in the Directorate for Geosciences. This year a Committee of Visitors (subsequently referred to as the COV) was asked to provide oversight on the operations of the Ocean Sciences Research Section (OSRS) of the Division of Ocean Sciences at NSF for the period 1992-1994. Most of the data evaluated were for FY94. In particular the COV was asked to evaluate the Biological Oceanography (BO) Program, the Chemical Oceanography (CO) Program, the Marine Geology and Geophysics (MGG) Program, the Physical Oceanography (PO) Program, and the Oceanographic Technology and Interdisciplinary Coordination (OTIC) Program. The latter is a program formed since the last COV. In addition to technology development, it has responsibility for activities that cut across all four basic science areas, such as the Coastal Ocean Processes (CoOP) initiative and the Arctic Systems Science (ARCSS) initiative. The program also has responsibility for some national and international community coordination and planning activities. The COV was also asked to review a small number of research proposals submitted annually to the Ocean Drilling Program for postdrilling activities that are reviewed by the MGG panel, and

Robert Duce. Texas A\&M University, College Station, TX 77843, USA. Sallie Chisholm, Massachusetts Institute of Technology, Cambridge, MA 02139. USA. Thomas Kinder, Office of Naval Research, Arlington. VA 22217. USA. Peter Liss. University of East Anglia. Norwich NR4 7TJ. United Kingdom. Ted Moore, University of Michigan, Ann Arbor, MI 48109, USA. Mary Scranton, State University of New York. Stony Brook, NY 11794. USA. some Education and Human Resources (EHR) proposals for which OCE has direct responsibility.

In addition to the areas outlined above, the COV decided to evaluate four other issues in more detail. These were:

Interdisciplinary proposals. The COV studied the statistics of such programs (i.e.. success rate, whether they are at a disadvantage within OCE, etc.) and how they are handled within OCE by subunits and by the OTIC Program. Note that this does not include the Large Science Programs (see below), many of which are interdisciplinary as well.

Large Science Programs. Large Science Programs (sometimes referred to as Global Change Programs) within OCE are organized through the $\mathrm{PO}, \mathrm{BO}, \mathrm{CO}$, MGG, and OTIC Programs. They include the World Ocean Circulation Experiment (WOCE) (PO), Tropical Oceans, Global Atmosphere (TOGA) (PO and the Division of Atmospheric Sciences at NSF), Joint Global Ocean Flux Study (JGOFS) (CO, BO), Global Ecosystems Dynamics (GLOBEC) (BO), Ridge Interdisciplinary Global Experiments (RIDGE) (MGG, BO), Earth System History (ESH, MGG), Land Margin Ecosystem Research (LMER, BO), and ARCSS and CoOP (OTIC). The COV tried to understand the process of how these programs are handled from beginning to end. Concerns were related to the level of "topdown" (i.e., NSF program manager) and steering committee (or others involved in laying out the programs) control over both funding decisions and how the program is carried out, and whether the individuals selected for funding are done so in a manner consistent with the agreed upon objectives and goals of the program plan.

Women, minorities, and young investigators. The COV examined the success rate of the funding of women, minorities, and young investigators compared with the overall pool of eligible scientists and the level of proactive activity in OCE to bring more women, minorities, and young investigators into oceanography and into the funding process.

Relationships between NSF and other agency funding. The COV examined how often NSF suggests that another agency is a more appropriate place for a proposal and what policies NSF has on which they base such suggestions; the extent to which NSF and other agencies co-fund research, particularly if one agency supported data collection and the other is asked to support scientific interpretation; and the general level of interaction and cooperation between program managers and higher administrators at NSF and other agencies that support ocean science.

Well before the $\mathrm{COV}$ meeting at NSF, the $\mathrm{COV}$ requested and obtained from NSF/OCE considerable information related to the areas that would be reviewed. During the COV meeting on 22-24 May 1995 at NSF, COV members met with individual program managers and associates as well as the OSRS Director to discuss these issues. The COV also had a plenary meeting with most of the program staff of the NSF Ocean Sciences Research Section to exchange information and ideas. COV members also evaluated a number of individual proposal jackets. In particular, the COV evaluated the integrity and efficiency of processes used to solicit, review, recommend, and document proposal actions and the relationship between award decisions and program goals. Also evaluated were the number of high quality reviewers and their apparent freedom from bias, and the balance of awards relative to subject mat- 
ter, size versus number of awards, and age experience and minority status of the investigators.

The COV wants to state strongly at the beginning of this report that the program managers and science associates in the OCE Ocean Sciences Research Section are doing an excellent job. They are clearly a dedicated group who are committed to the wise use of NSF funds to support the best possible science. They are also committed to making accurate and unbiased decisions based on objective data and reviews.

\section{Review of OSRS Programs}

Members of the COV inspected a representative number of proposal jackets in each of the six programs that were evaluated. Proposal jackets for review were generally selected based on one of three criteria-poorly rated proposals that were funded, well-rated proposals that were declined, and proposals where the average panel review score differed significantly from the average mail review score. In some cases proposal jackets were chosen at random.

During the period 1983-1994 for which we had uniform data, the number of proposals submitted annually increased from $\sim 800$ to $>1,100$ for the OSRS. Over the same period, success rates fell from $\sim 40$ to $29 \%$. We note with concern that 1994 was the worst year in the period. with the highest number of proposals $(1,147)$ and the lowest success rate $(29 \%)$. If the recent trend continues, both the ocean science community and NSF will be challenged to cope with increasingly sparse funding relative to community proposal pressure.

In subsequent sections of the report. we use the following terminology: "Core programs" refers to individual investigator unsolicited research proposals; "Large Science Programs" refers to multiinvestigator, often multidisciplinary, highly organized research efforts such as WOCE, JGOFS. RIDGE, GLOBEC, etc.; and "OSRS disciplinary programs" refers to a combination of core programs and Large Science Programs funded in the PO, BO, $\mathrm{CO}$, and MGG programs.

\section{Comparison of Programs in the Ocean Sciences Research Section}

\section{Long-term Growth}

Since 1986 the budget for OSRS disciplinary programs (including both core funding and the Large Science Program funding for those programs) has grown from 56.9 to 91.8 million dollars. Figure 1a shows the total funding for each of the OSRS disciplinary programs (i.e., $\mathrm{BO}, \mathrm{PO}, \mathrm{CO}$, and $\mathrm{MGG}$ ) in current (actual, no inflation factor applied) dollars, and Figure $1 \mathrm{~b}$ shows the overall funding for OSRS in both current and FY 1994 dollars. Table 1 shows the total funding as well as the breakdown between core funding and Large Science Program funding for the four disciplinary programs. Most of the $61 \%$ growth has come with the addition of the Large Science Programs. These large, multiinves-

\section{Ocean Sciences Research Section FY 83-95 Program Budgets (Current \$)}
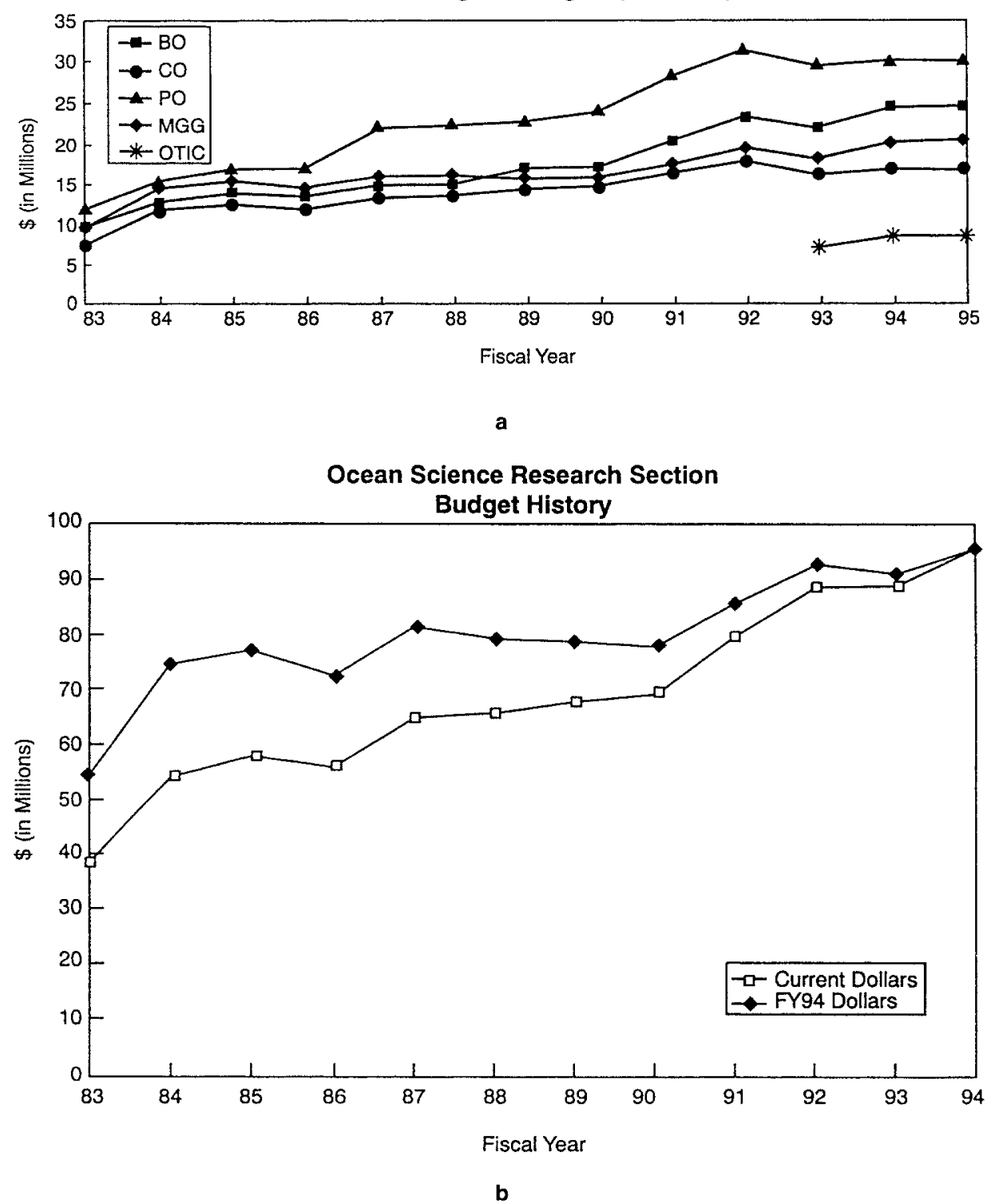

Fig. 1: (a) Budgets of OSRS research programs from FY 1983 to FY 1995 in current (actual, no inflation factor applied) dollars. (b) Total budget for OSRS in current (actual, no inflation factor applied) and FY 1994 dollars. 
Table 1

Comparison of program budgets, current dollars (millions of dollars)

\begin{tabular}{|c|c|c|c|c|c|}
\hline & $\mathrm{PO}$ & BO & MGG & $\mathrm{CO}$ & Total \\
\hline \multicolumn{6}{|c|}{ Total OSRS Disciplinary Program Budgets } \\
\hline FY 1986 & 17.1 & 13.3 & 14.6 & 11.9 & 56.9 \\
\hline FY 1994 & 29.9 & 24.3 & 20.6 & 17.0 & 91.8 \\
\hline \multicolumn{6}{|c|}{ Core Programs } \\
\hline FY $1986^{*}$ & 17.1 & 13.3 & 14.6 & 11.9 & 56.9 \\
\hline FY 1994 & 13.8 & 13.5 & 14.7 & 12.0 & 54.8 \\
\hline \multicolumn{6}{|l|}{$\begin{array}{l}\text { Percent change } \\
\text { in core FY }\end{array}$} \\
\hline $1986-1994$ & -19.3 & +1.5 & +0.7 & +0.8 & -3.7 \\
\hline \multicolumn{6}{|c|}{ Large Science Programs, FY 1994} \\
\hline WOCE & 11.9 & & & & 11.1 \\
\hline TOGA & 4.2 & & & & 4.2 \\
\hline JGOFS & & 5.0 & & 5.0 & 10.0 \\
\hline RIDGE & & 0.9 & 4.9 & & 5.8 \\
\hline GLOBEC & & 3.8 & & & 3.8 \\
\hline LMER & & 1.1 & & & 1.1 \\
\hline $\mathrm{ESH} \dagger$ & & & 1.0 & & 1.0 \\
\hline Totals & 16.1 & 10.8 & 5.9 & 5.0 & 37.0 \\
\hline
\end{tabular}

* Note-Large Science Programs began in FY 1987. Thus all the budget figures for FY 1986 refer to the core program at that time. $\uparrow$ OCE funded aspects of ESH, sometimes referred to as MESH.
Science Program research money is presently focused in $\mathrm{PO}$ and $\mathrm{BO}$.

As the Large Science Programs have grown, the amount of research money available in the core programs has, on the average, decreased. If inflation is considered, funding for core programs has decreased significantly since FY 1986 (Table 1). The amount of research money assigned in FY 1994 to each OSRS disciplinary program to fund these single investigator, unsolicited proposals is fairly evenly distributed among the OSRS programs, ranging from about $\$ 12$ to $\$ 15$ million (Table 1). Thus the total research dollars (core programs plus Large Science Programs) spent in the OSRS disciplinary programs in 1994 is also highest in $\mathrm{PO}$ and $\mathrm{BO}$.

The number of awards made within the OSRS disciplinary programs has, on occasion, varied by $>30 \%$ from one year to the next. However, three of the four OSRS disciplinary programs have had some slight long-term average growth in the number of awards over the past 11 years (as determined by linear regression of the data in Fig. 3). The number of awards in $\mathrm{CO}$ has not shown such a growth trend. The median size of the awards increased for most programs over the past 11 years when considered as "current" dollars (unadjusted for inflation, as shown in Fig. 4a). However, when the award size is ad- number of $\mathrm{CO}$ proposals has grown very little in the last 5 years (1989-1994). The number of proposals submitted to the PO and $\mathrm{BO}$ programs have grown by very modest average growth rates of $\sim 5-6 \%$. However, BO has shown extremely wide swings, with as few as $\sim 230$ proposals submitted in 1983 and 1988 and nearly 400 proposals submitted in 1985 and 1992. MGG has had a higher and more steady long-term average growth rate in the number of proposals submitted. $\sim 15 \%$ per year.

It is obvious from these figures that as proposal demand has grown at a relatively high rate and awards have grown at a much lower rate, the average success rate for proposals has declined (Fig. 6). In 1994 the success rate of all OSRS disciplinary proposals (core programs and Large Science Program combined) submitted ranged between 26 and 35\%, with $\mathrm{BO}$ having the lowest success rate and PO the highest (Table 2, Fig. 6).

The increased proposal pressure has occurred at a time when OSRS has made a conscious effort to increase the average duration of awards, and they have been successful (Fig. 7). This effort was aimed at decreasing the amount of time that scientists needed to spend applying for and evaluating proposals relative to the amount of time that they could do research. As a result, over the past decade the mean award duration has increased from $\sim 2$ yr to $2.3 \mathrm{yr}$ for MGG and from 2.4 to almost 3 yr for PO.

\section{Panel and Mail Reviews}

For all of the OSRS disciplinary programs (core programs plus Large Science

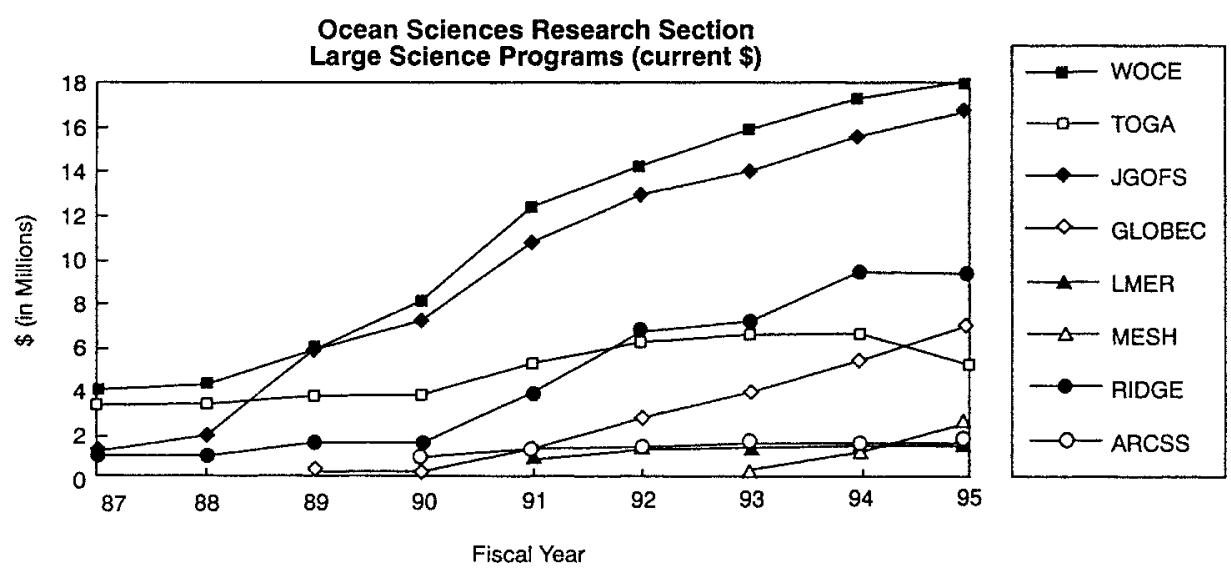

Fig. 2: Budgets of OSRS Large Science Programs from FY 1987 to FY 1995 in current (actual, no inflation factor applied) dollars. These figures include ship operating funds. $M E S H$ refers to the aspects of ESH funded by OCE. 


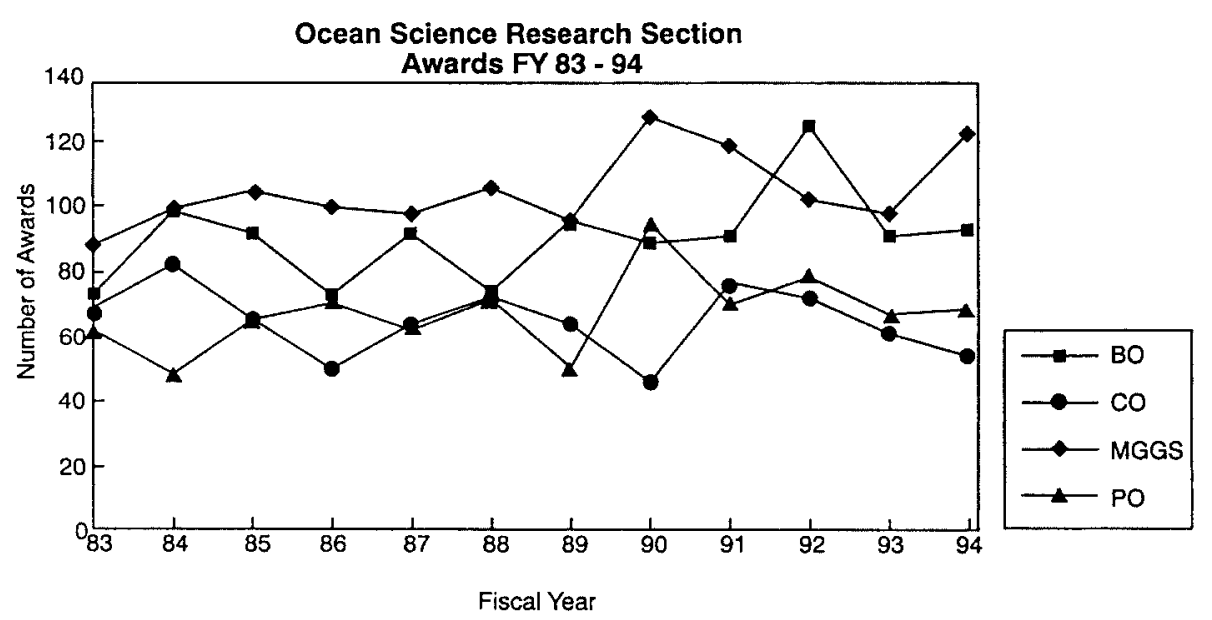

Fig. 3: Number of awards in OSRS disciplinary programs from FY 1983 to FY 1994.
Programs) in FY 1994, the mean of the review scores given proposals by a review panel are poorer than the mean of the mail reviews, although the differences do not appear to be statistically significant (Table 3). This probably reflects the

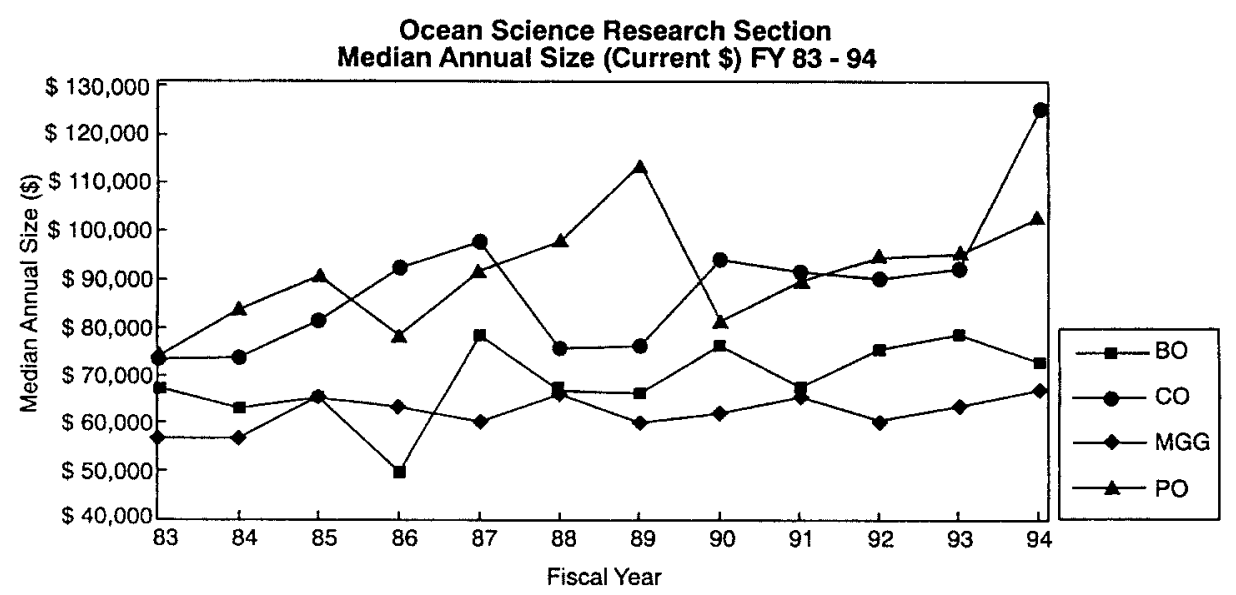

a

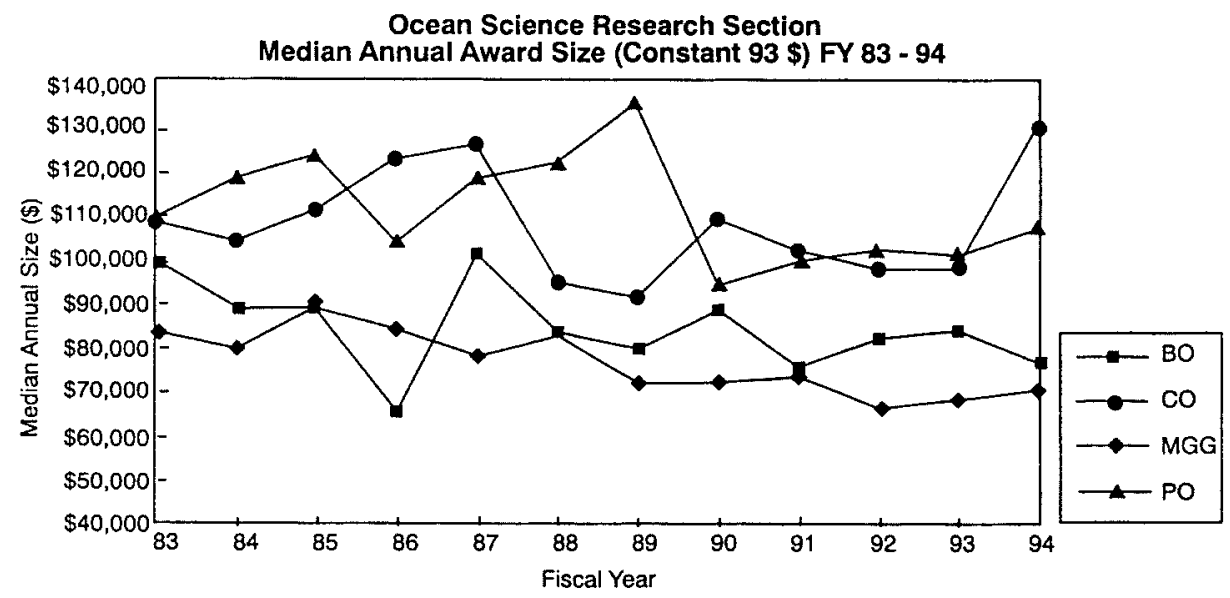

b

Fig. 4: (a) Median annual funding for individual grants in OSRS disciplinary programs from FY 1983 to FY 1994 in current (actual, no inflation factor applied) dollars. (b) Median annual funding for individual grants in OSRS disciplinary programs from $F Y$ 1983 to FY 1994 in constant FY 1993 dollars.
Table 2

OSRS mean panel scores and proposal success rates. FY 1994

\begin{tabular}{cccc}
\hline & Mean & Number & \\
Panel & of & Success, \\
Program* & Score & Proposals Awards & $\%$ \\
\hline
\end{tabular}

Core Programs

\begin{tabular}{lrrrr} 
BO & 2.4 & 360 & 92 & 26 \\
CO & 2.5 & 167 & 53 & 32 \\
PO & 2.7 & 193 & 68 & 35 \\
MGG & 2.3 & 427 & 122 & 29 \\
Total & & 1.147 & 335 & 29 \\
\hline
\end{tabular}

Scores: 1 = Excellent, 4 = Poor. * Includes both Large Science Program and core program projects.

fact that the panels are privy to all the criticisms and evaluations of each proposal made by mail reviewers and are also charged with conducting their own review, integrating this information, and ranking the proposals received. There is, however, a positive correlation between the scores given by the panels and mail reviewers. Although there is considerable scatter in these relationships (Fig. 8, a-d). this scatter appears unrelated to either the average chance of success of a proposal or to the average panel score (cf., Fig. 6-8 and Table 2).

Average panel scores for the three largest Large Science Programs have a much broader spread and tend to be better than for the OSRS disciplinary programs as a whole (Fig. 9-the average panel review scores indicated in this figure refer to all proposals submitted that were rated by the panels, not just funded proposals). The better panel scores may result from the more focused, carefully planned, field-work intensive, and integrated nature of the proposed research in these larger programs. As a result of these factors, many of the individual proposals may be highly interdependent and important to the success of the program as a whole. Success rates in these larger programs tended to be higher than those of the overall OSRS disciplinary programs in FY 1994. Proposals submitted for consideration as part of a large program are often planned or scrutinized for relevancy to the program in question. Thus, in effect they have a pre-review that may in some cases result in a statistical bias in the number, score, and success rate of the Large Science Program proposals when comparing them with the core program proposals. However. a comparatively large number of proposals were submitted to RIDGE in 1994, and it 


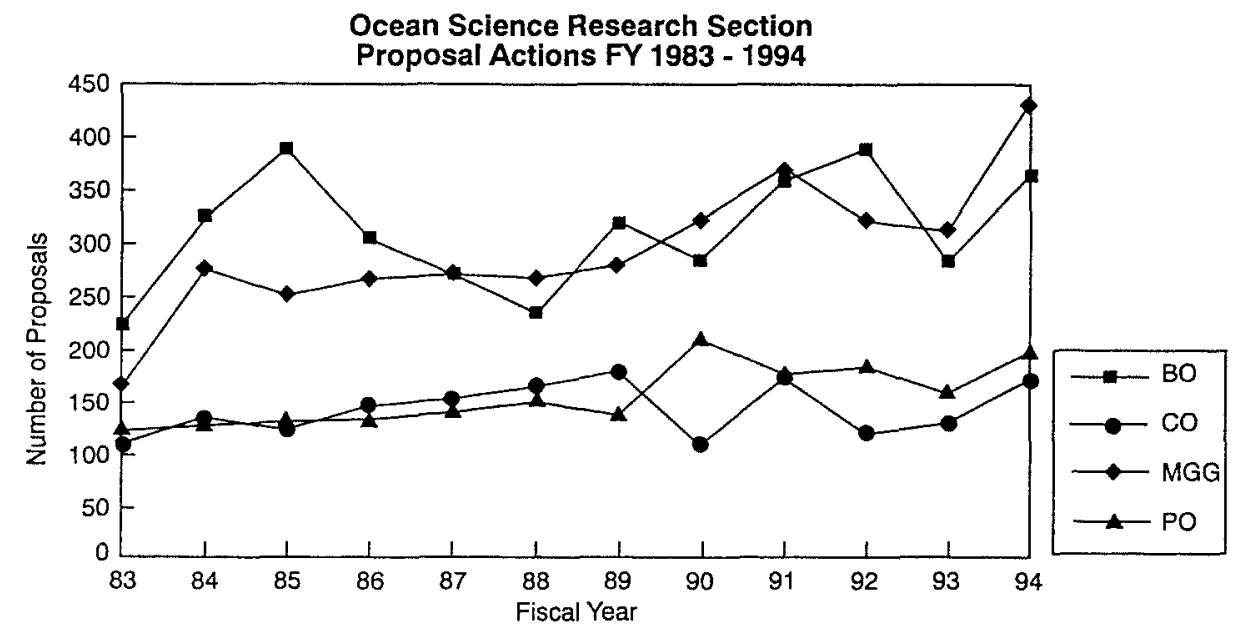

Fig. 5: Number of proposals submitted to OSRS disciplinary programs from FY 1983 to $\mathrm{FY} 1994$.

had a success rate that was only slightly higher than that of the MGG core programs in OSRS.

\section{Review of Specific Programs}

Biological Oceanography Program (BO)

The Biological Oceanography program received 360 proposals to consider in 1994; 92 were awarded, representing a success rate of $26 \%$. In 1993 it received 275 proposals and awarded $86(31 \%$ success rate), and in 1992 it received 380 proposals, awarding $118(31 \%$ success rate). The success rate of $\mathrm{BO}$ proposals has remained at a constant of roughly 30 $\pm 5 \%$ for over a decade. The other programs have traditionally enjoyed higher success rates than this, but they now appear to be converging at this level (Fig. 6). MGG in particular has been at this level for the last 3 years. The low success rates of $\mathrm{BO}$ and $\mathrm{MGG}$ emerge from higher than average numbers of applicants, in spite of higher numbers of awards. The average award size in $\mathrm{BO}$ has decreased roughly $\$ 20,000$ (constant $93 \$$ ) in the last decade, settling in at $\sim \$ 80,000$ in 1993 . Only MGG has lower mean award sizes.

Twenty-five proposal jackets were examined in detail. Upon careful examination of the documentation in each jacket, most importantly the summary of the panel deliberations, we found no problems with the rationale behind the decisions. In one case it was observed that a young investigator was twice declined for a highly ranked proposal submitted for core funds, and funded the first time for a lower ranked proposal submitted to one 1983 to FY 1994. years, although the overall trend has been for rates to decrease. Most recently, in FY 1992 the success rate exceeded 60\% [apparently due to the EqPac (Equatorial Pacific) Program], whereas in FY 1994 the success rate was the lowest it has been since data have been maintained in the computer. At present the success rate for $\mathrm{CO}$ is about the same as for the other programs.

In FY $1994 \mathrm{CO}$ awarded 53 grants and declined 114 proposals, with an overall success rate of $32 \%$. Eighty-nine percent were rated by a panel as well as by mail reviewers. Fourteen proposals related to the Arabian Sea JGOFS program were funded, and 31 Arabian Sea JGOFS proposals were declined (giving a success rate for JGOFS for these proposals of $31 \%$ ). The overall success rate for JGOFS was higher, however, $\sim 50 \%$. Of the declined Arabian Sea proposals, seven were for rain and aerosol-related research, and eight were sediment related. We were informed by the program manager that the latter groups were declined because of insufficient funds and the program's decision, based on JGOFS' identification of essential core measurements in the solicitation announcement, that rain, aerosol, and sediment proposals were of lower priority.

Most of the proposals funded had both mail and panel mean scores better than or equal to 2.0. Most of those funded outside of this range received mail scores higher than 2.5 but were given better scores by the panel. Most of these were for important JGOFS measurements.

Twenty proposal jackets were selected for examination. One jacket could not be

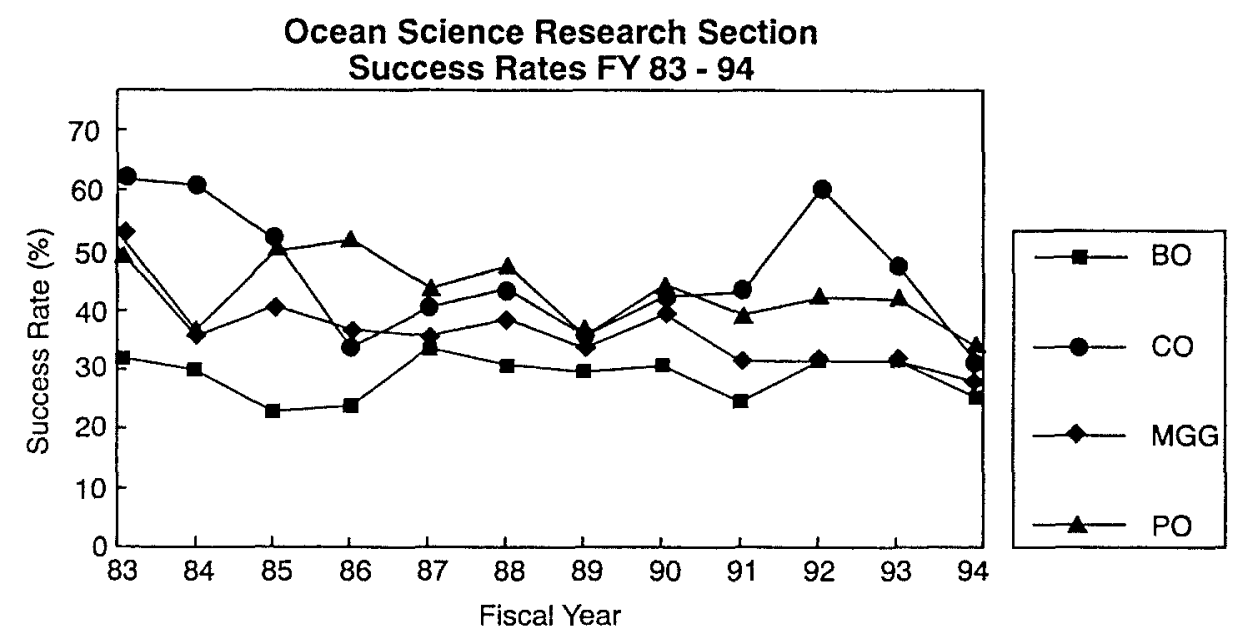

Fig. 6: Success rate for proposals submitted to OSRS disciplinary programs from $F Y$ 


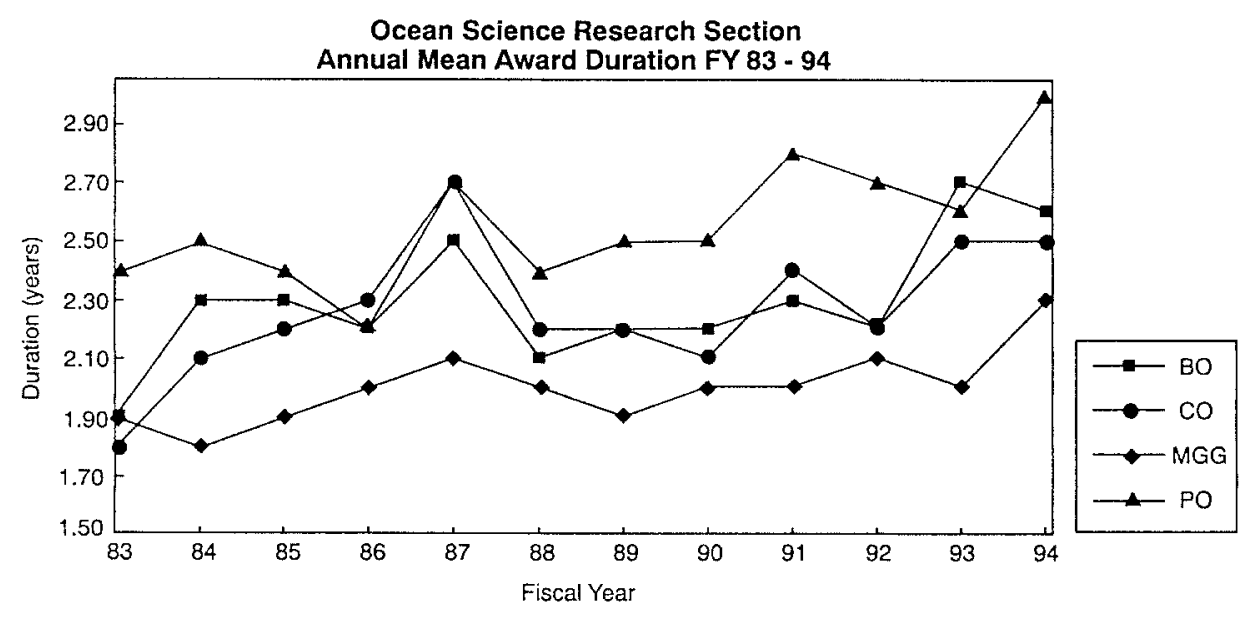

Fig. 7: Mean award duration for proposals funded by OSRS disciplinary programs from FY 1983 to FY 1994.

located. A number of the funded proposals with poorer ratings were for "service" or "management" efforts associated with core measurements for the Arabian Sea. In most of these cases, reviewers and panelists acknowledged the central nature of the measurements in these proposals, but nonetheless tended to give them poorer scores. The remainder of the proposals funded in spite of weaker scores contained both well rated and poorly rated components. In these cases, funding was at reduced levels for the strong portions of the proposal. One proposal submitted for the Arabian Sea was funded from the core program because of enthusiasm for the basic science in spite of weak ratings for JGOFS relevance.

We also inspected a number of files for proposals that had high ratings but were declined. There were a variety of explanations for such decisions, including rejection of companion proposals and letter review text as well as panel discussions that were measurably more critical than the numerical ratings assigned. Three files were inspected in which the proposal was rated fully fundable but the proposal was declined due to lack of funds.

Finally, we inspected a number of files for which panel scores and mail review scores differed by more than a point. In all cases, the panel score mean was poorer than the mail review score mean. Some of these proposals were JGOFS proposals rated less highly by the panel for not satisfying JGOFS goals. Others were cases in which panelists were strongly influenced by critical comments of mail reviewers. All decisions examined seemed adequately justified, although the COV members might not have made the same decision in all cases.

\section{Marine Geology and Geophysics Program ( $M G G$ )}

The success rate of MGG proposals has gradually declined since 1990, and in 1994 it reached an all-time low of $29 \%$ (Fig. 6). At the same time, the number of proposals reviewed by the MGG program reached an all time high of 427 . These changes have occurred at a time when the overall funds in the MGG program have grown only slightly in current dollars (unadjusted for inflation, Fig. 1a).

The MGG panel rated $>40 \%$ of all proposals submitted to that section. Those proposals not rated by the panel tended to have either very fine or very weak mail review rankings and were either awarded or declined on the basis of these mail reviews. The plot of the mail and panel numerical scores for each proposal shows a very broad scatter (Fig. 8d). with a very loose positive correlation. Mean mail review scores tend to be better than those assigned by the panel.

Thirty-five FY 1994 proposals were selected for review by the COV. All of the proposals selected had at least three mail reviews. The written documentation for all awards and declines was found to be excellent. Declined proposals that ranked relatively well were usually declined based on comments made by mail and panel reviewers that identified specific flaws in the proposals. Occasionally proposals were declined based on relative costs and programmatic balance. Less highly ranked proposals that were funded often constituted a critical part of a larger program (such as RIDGE). The need for these particular elements in the program was clearly documented. The final decision to fund or decline a proposal lies with the program manager, and it appears that the program managers have exercised this power judiciously for the good of the overall program. The bases for all decisions made were well documented.

Physical Oceanography Program (PO)

The Physical Oceanography disciplinary program considered 193 proposals in FY 1994 and made 68 awards $(35 \%$ success rate). Since FY 1983, the number of proposals submitted to PO has risen from $\sim 130$, and the success rate has declined from $\sim 45$ to $<40 \%$ (FY 1994 had the 2 nd highest number of submissions and the lowest success rate in the period). Most proposals were reviewed by panels (as evidenced by panel scores-panels may discuss proposals, but decide not to review them formally). In FY 1994 there were 12 awards that were not reviewed by a panel and 22 declinations that were not reviewed.

Forty-three proposal jackets were reviewed by the COV. One requested jacket could not be located. All decisions that appeared anomalous were examined: less highly ranked proposals that were funded, more highly ranked proposals that were declined, or proposals with a wide separation between the mean mail and panel review scores. All of the proposal files were complete and contained sufficient documentation to understand the decision of the program manager. All apparent anomalies could

\section{Table 3}

Mean panel and mail review scores by overall OSRS disciplinary program. FY 1994

\begin{tabular}{lcc}
\hline Program*s & $\begin{array}{c}\text { Mean Panel } \\
\text { Review Score } \pm s\end{array}$ & $\begin{array}{c}\text { Mean Mail } \\
\text { Review Score } \pm\end{array}$ \\
& \multicolumn{3}{c}{ Awards } \\
BO & $1.8 \pm 0.6$ & $1.8 \pm 0.4$ \\
CO & $2.2 \pm 0.7$ & $2.0 \pm 0.5$ \\
PO & $2.1 \pm 0.6$ & $1.9 \pm 0 \pm$ \\
MGG & $1.9 \pm 0.4$ & $1.7 \pm 0.4$ \\
& & \\
& Dectines & \\
BO & $2.6 \pm 0.6$ & $2.4 \pm 0.6$ \\
CO & $2.7 \pm 0.6$ & $2.4 \pm 0.5$ \\
PO & $3.0 \pm 0.5$ & $2.5 \pm 0.5$ \\
MGG & $2.5 \pm 0.5$ & $2.4 \pm 0.6$ \\
\hline
\end{tabular}

Scores: $1=$ Excellent $4=$ Poor. 


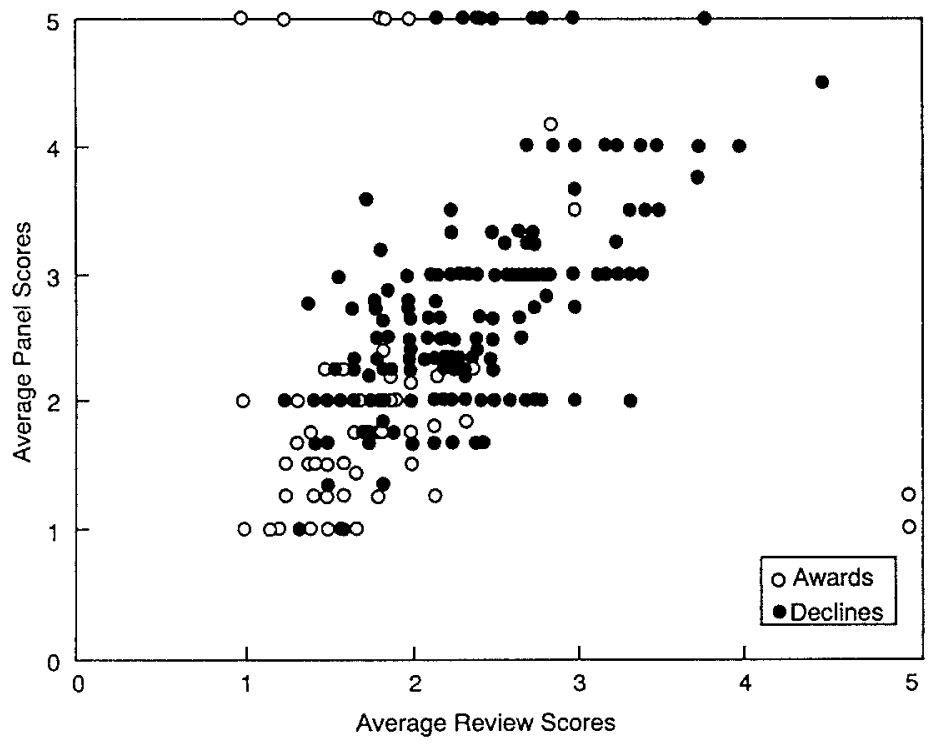

a

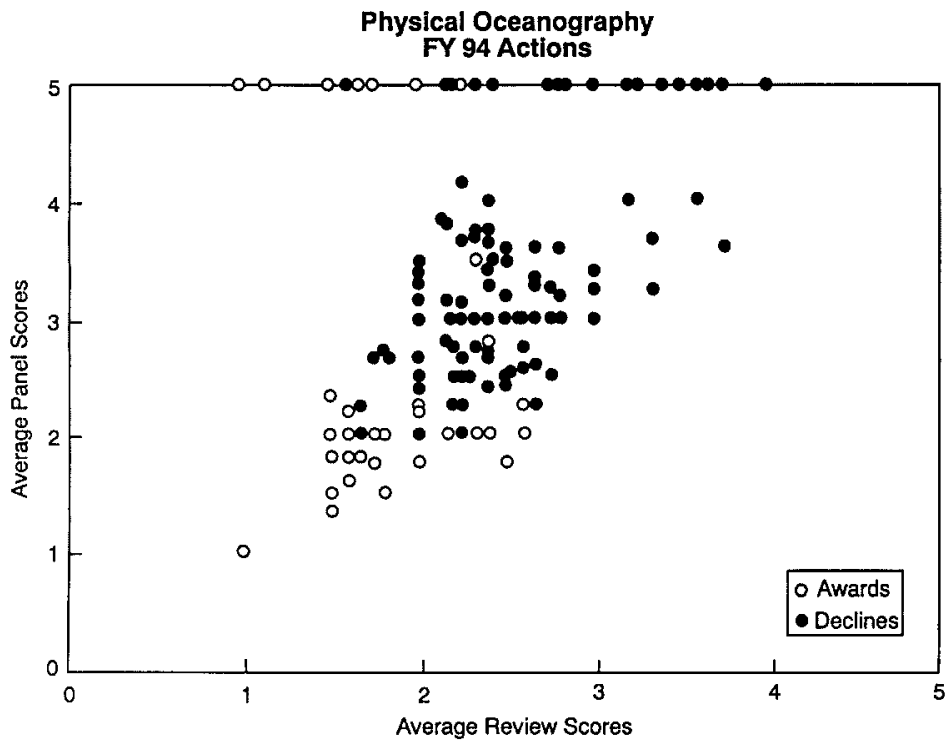

c

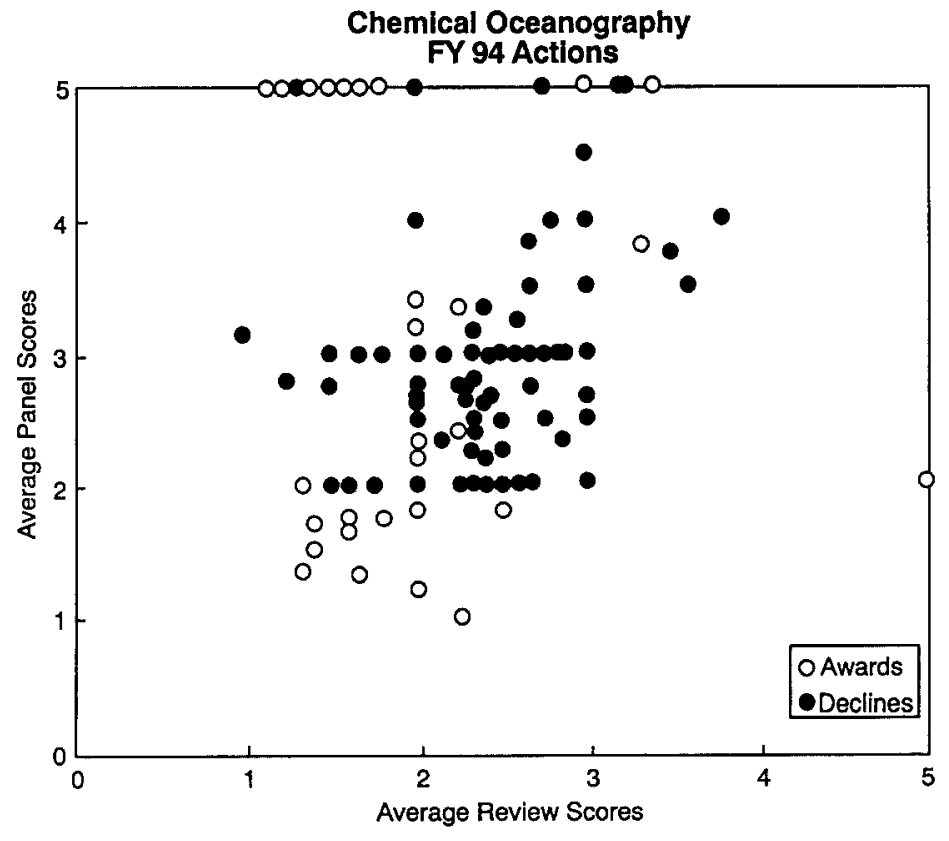

b

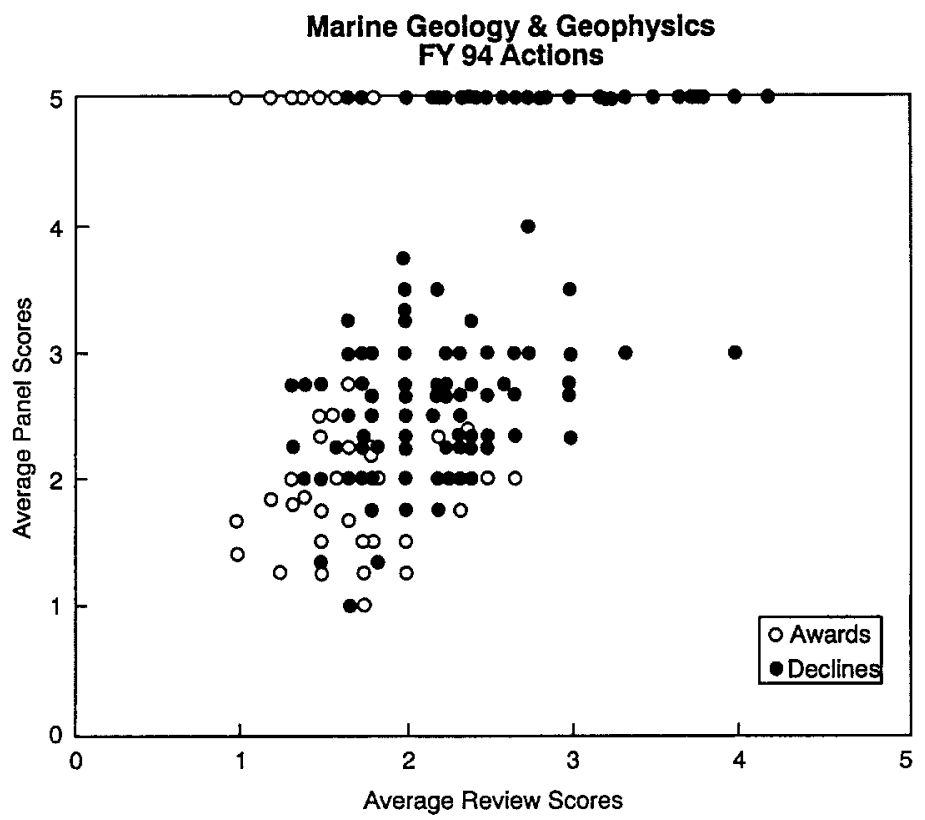

d

Fig. 8: (a) Average panel scores versus average mail review scores for awards and declines for BO (Score $1=$ Excellent; Score $4=$ Poor). A panel score of 5 was given to those proposals not reviewed by the BO panel. (b) Average panel scores versus average mail review scores for awards and declines for CO (Score I = Excellent; Score $4=$ Poor). A panel score of 5 was given to those proposals not reviewed by the $C O$ panel. (c) Average panel scores versus average mail review scores for awards and declines for $P O$ (Score $1=$ Excellent: Score $4=$ Poor). A panel score of 5 was given to those proposals not reviewed by the PO panel. (d) Average panel scores versus average mail review scores for awards and declines for MGG (Score $1=$ Excellent; Score $4=P$ Por). A panel score of 5 was given to those proposals not reviewed by the MGG panel.

be rationalized from the documentation. The reasons for the anomalies fell into two general categories: action by the review panel and action by the program manager.

A common anomaly for declined proposals was a more highly ranked pro- posal from mail reviewers, but less highly ranked by the panel. Panels pay close attention to the written comments of mail reviewers, and they attempt to understand the basis for the scores. There were cases where superficial mail reviews gave good scores, or where mail reviewers gave good marks based on the previous work of the proposer, but were skeptical about the merit of the actual proposal. In both situations, panelists recognized that the mail reviews, in spite of being more highly ranked, did not support funding the proposals. 


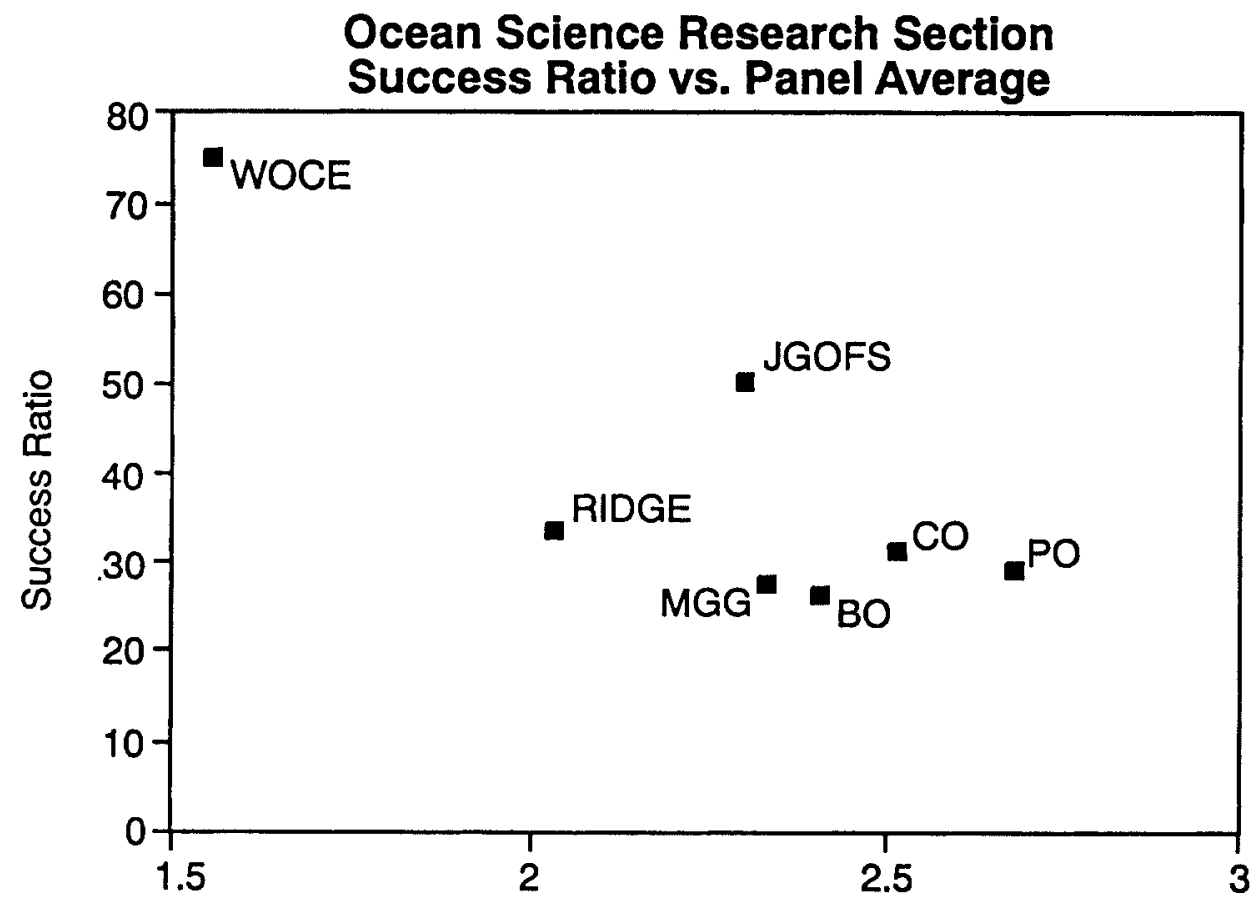

Average Panel Review

Fig. 9: Success ratio versus average panel review score for the larger OSRS Large Science Programs and the OSRS disciplinary programs in FY 1994.

There was one case where a large international and multiinvestigator project was funded in spite of weak mail and panel reviews. In this case the mail reviews were biased by one particularly strongly stated review that greatly influenced the panel. The proposers offered the program manager a cogent point-by-point rebuttal of the strongly stated review and decreased the requested funding. Additionally, an international partner offered substantial cost sharing. The program manager carefully documented the reviews and the decision to fund. This funding decision was the strongest example of program manager intervention found in the files, and it was done carefully and with good reason.

In summary, raw scores are a crude measure of the merit. Both panelists and program managers focus on the written content of reviews. Program managers in PO appropriately examined the review processes and used the written comments and discussion to reach their decisions.

\section{Oceanographic Technology and Interdisciplinary Coordination (OTIC) Program}

The COV inspected 10 proposal jackets in the Oceanographic Technology and proximate funding ratio of 40:60. Although this might seem a somewhat untidy administrative arrangement, it appears to work satisfactorily and does provide some advantages in terms of flexibility of funding.

The funds for International/Interagency activities are effectively taken off the top of the central OCE allocation. This policy should be continued because it avoids direct competition between support for such activities and applications for individual research grants.

\section{Education and Human Resources Proposals}

The Ocean Sciences Division has responsibility for a number of proposals in the area of education and human resources. This area covers Research Experience for Undergraduates (REU) site proposals, proposals for minority activities in the ocean sciences, and NSF Young Investigator (NYI) proposals. Ten proposal jackets were evaluated in these areas. A mixture of funded and unfunded proposals was evaluated. and there were no unusual situations in the 10 proposal jackets selected for evaluation.

In FY 1994 nine proposals were submitted in the REU and minorities category, and eight were funded. All four of the REU proposals submitted were supported. Of the five proposals for support of minority undergraduate students, four were supported. Active efforts were clearly being made to encourage strong proposals in the REU and minority programs, and these areas appeared to be well managed.

During this same time period, $12 \mathrm{NYI}$ proposals were submitted, and 4 were funded. NYI proposals are reviewed by a directorate-wide panel that includes OCE input. Proposals to be funded are then transferred to the appropriate disciplinary program (together with funds) for processing. During FY 1994 there were two NYI proposals funded in $\mathrm{PO}$, one in $\mathrm{BO}$. and one in MGG. (In FY 1993 there was 1 from $\mathrm{PO}$ and 1 from $\mathrm{BO}$ funded.)

\section{Review of Special Issues}

\section{Interdisciplinary Proposals}

Interdisciplinary proposals can involve more than one subject within OCE (e.g., biology and physics) or span across divisions (e.g., oceans and atmosphere). They are important because scientific advance is often made at the intersection of tradi- 
tional disciplines. Often interdisciplinary proposals go to Large Science Programs (many of which are intrinsically interdisciplinary) or OTIC, which are dealt with elsewhere in this report. Here we are concerned with individual interdisciplinary proposals. These receive special treatment in the review procedure, and it is necessary for this to be as transparent as possible because there is a perception in the community that interdisciplinary proposals may "fall between the cracks" between traditional administrative divisions.

Although one program takes the lead in administering interdisciplinary applications, the relevant program managers jointly select mail reviewers where necessary. When supported, the funding of an interdisciplinary proposal will involve more than one program/division, by internal NSF definition.

Inspection of funding results and individual jackets revealed no obvious bias, one way or the other. in the treatment of interdisciplinary proposals relative to monodisciplinary proposals. The jacket documentation may be somewhat incomplete in that it does not always include information on much of the negotiation, which is by conversation between program managers. This tends not to be recorded. One program manager expressed the view that any problems were more likely to be between NSF divisions, rather than across programs within OCE.

It does not seem sensible to have a special allocation of funds for interdisciplinary proposals (other than in OTIC and some Large Science Programs). This is in part because any problems with the present arrangements do not appear to be large, but also because the rather small number of such proposals would not justify the extra administrative burden involved.

\section{Large Science Programs}

As was the case three years ago, the Large Science Programs within NSF have received the bulk of the new funding that has come to the Geosciences Directorate in recent years. This has been the case for funding for the Ocean Sciences Research Section as well. The rest of the individually funded programs (the core programs) have not kept up with inflation. In fact, as has been observed by other COVs, the increases in funding lev- els of the Large Science Programs have corresponded to decreases in core funding (in terms of constant dollars) in the last decade. There is no reason to believe that the situation will change in the near future, and even funding for global change research at increased or even the same level is not assured.

The total OSRS budget increased from $\$ 57$ to $\$ 92$ million between 1986 and 1994 (Table 1), all of this increase going into the Large Science Programsprimarily WOCE and JGOFS, but including TOGA, RIDGE, GLOBEC, LMER. and the marine component of ESH. Although the funding from these programs goes in general to investigators who would otherwise be funded out of core funds, there is also no doubt that these programs have changed the character of research in oceanography, and if growth continues in this direction without a concomitant increase in core funding it could change fundamentally the way we do our science. To proceed in this direction without trying to understand the longterm consequences would be imprudent.

Both the scientific community and the NSF staff hope that when the Large Science Programs "wind down," the elevated funding levels enjoyed by these programs will remain for the core (or another initiative that could spin up)as was the case for programs in the International Decade of Ocean Exploration (IDOE). This represents a strong argument for keeping the Large Science Programs healthy, and a compelling positive dimension. However. there are no guarantees that this will happen. In addition, there apparently are few "sunset clauses" in the science plans for any of the Large Science Programs, so there is often no clear mechanism in place to ensure that the balance between core programs and Large Science Programs will not continue to tilt toward the latter.

Although there is some variation in the way the different Large Science Programs are run, all but RIDGE have a process that is a bit tangential to the regular review and panel process. The operational structure of the RIDGE program-i.e., the handling of proposals through the regular panel-seemed to us to represent a healthy compromise in the "quality versus coordination" trade-offs that are inherent in these Large Science Programs.

\section{Women, Minorities and Young} Investigators

Previous COV reports have examined the success rates for women and for scientists who have received their $\mathrm{PhDs}$ in the last 5 years. The previous COV indicated that, in FY 1991, women were almost exactly as successful as the general population. For FY 1994 applications from women constituted $\sim 19,10,9$, and $15 \%$ of total applications for $\mathrm{BO}, \mathrm{CO}$, PO. and MGG, respectively, whereas applications from recent PhDs were $\sim 19$, 13,18 , and $25 \%$, respectively, of total applications. Information for success rates in FY 1994 is presented in Table 4, and it shows the same pattern as has been seen by previous COVs. Differences in success rates for women from the success rate for the whole population are likely to be due to small sample sizes in $\mathrm{PO}$ and $\mathrm{CO}$, as is the high success rate for recent PhDs in CO.

The COV also discussed the status of underrepresented groups among NSF OCE proposers and awardees. Based on statistics provided by NSF, the number of applications by members of underrepresented groups is quite low, so statistics on success rates are of questionable value. However, over the period FY 1993-1994, Hispanic PIs had roughly the same success rate as the general population $(28 \%)$, whereas no proposals by African-American PIs were funded (see Table 5).

In discussion with the COV, program managers were generally aware of the identity of applicants from underrepresented groups. However, it appeared that ethnic status played little or no role in whether a proposal was funded or not. The lack of proposal pressure from underrepresented groups is generally perceived to be a result of problems with the "pipeline" or supply of individuals from these groups who are coming through the undergraduate and graduate ranks. However, there is also no formal follow-up within OCE to assist PIs from underrepresented groups, although the science associate in charge of minority programs attempts to fill this role. Because individuals from nontraditional backgrounds may be less familiar than other individuals with how best to take advantage of the opportunities that exist within NSF and elsewhere in the science community. such an active role might help to improve the poor showing of scientists from un- 
Table 4

Success Rates for Women and Recent PhDs. FY 1994

\begin{tabular}{|c|c|c|c|c|c|c|}
\hline \multirow[b]{2}{*}{ Program } & \multicolumn{2}{|c|}{ All PIs } & \multicolumn{2}{|c|}{ Women } & \multicolumn{2}{|c|}{ Recent PhDs } \\
\hline & $\begin{array}{l}\text { Number } \\
\text { of Awards }\end{array}$ & Success, \% & $\begin{array}{l}\text { Number } \\
\text { of Awards }\end{array}$ & Success. $\%$ & $\begin{array}{l}\text { Number } \\
\text { of Awards }\end{array}$ & Success. \% \\
\hline $\mathrm{BO}$ & 92 & 26 & 21 & 31 & 21 & 31 \\
\hline $\mathrm{CO}$ & 53 & 32 & 9 & 53 & 11 & 50) \\
\hline $\mathrm{PO}$ & 68 & 35 & 4 & 22 & 12 & 34 \\
\hline MGG & 122 & 29 & 20) & 31 & 25 & 24 \\
\hline
\end{tabular}

derrepresented groups in the pool of NSF PIs. The DISCO (Dissertations Symposium in Chemical Oceanography) and DIALOG (Dissertation Abstracts in Limnology and Oceanography) programs, sponsored in part by NSF, have gone a long way in demystifying the funding process for young scientists, and perhaps efforts could be made to include individuals with nontraditional background or those from smaller institutions where knowledge of the funding process is less ingrained. [Efforts of this sort are common in programs like the EHR Instrumentation for Laboratory Improvement (ILI) Program, which could be contacted for advice.]

There are several programs within OCE to increase the pipeline by involving a number of people from underrepresented groups in oceanography at an earlier stage in their career. These include programs for minority undergraduate and graduate students, and a number of general REU sites that are encouraged to recruit and admit minority undergraduates. These have been discussed earlier in the report. The science associate in charge of these programs is working with the REU sites to improve communication between the sites and to enhance longitudinal record keeping so that the careers of REU participants can be monitored. This is a very positive step. The next COV should be in a position to better evaluate the success of such efforts in recruitment and training of students from underrepresented groups. as the first cohorts of students from these programs will have begun to complete their graduate degrees.

\section{Relationships Between NSF and Other Agency Funding}

Interactions with other agencies can enable ambitious research programs and the efficient use of scarce funding. At the same time, the interactions between multiple agencies and their review processes can lead to detrimental results. such as biases that impede merit-based proposal evaluation. There are three important categories of NSF interaction with other agencies: large projects, individual proposals submitted to NSF without explicit links to other agencies, and proposals submitted to NSF that are associated with other agencies.

NSF has strong collaboration with other agencies in many Large Science Programs, such as JGOFS, WOCE, GLOBEC, and TOGA. Although there is debate about the wisdom of some large projects and their effects on science and science funding (i.e., "small" science versus "big" science), the interagency aspects of these programs work well. The collaborations benefit the researchers, the mission agency (e.g.. Office of Naval Research, National Oceanic and Atmospheric Administration, National Aeronautics and Space Administration. Department of Energy), and NSF.

NSF program managers actively seek joint funding of meritorious individual proposals that are potentially interesting to mission agencies. Such joint funding keeps mission agencies aware of relevant research and provides natural links between NSF and the mission agencies at the program manager level. The mission agencies, in effect, take advantage of the NSF review system, and the NSF is able to stretch limited funding.

The third category arises when individual proposals have connections to other agencies that may affect how reviewers judge them. Examples include proposals previously declined by another agency, proposals to finish work begun with other agency funding, or proposed work that may be perceived as "belonging" to another agency ("Why doesn"t DOE fund this proposal?"). The NSF program managers seem sensitive to biases that may influence the reviews of such proposals, and they are active in compensating for such biases and ensuring that reviewers judge proposals on merit. There is insufficient documentation to assess objectively how well "other agency" biases are handled.

\section{Conclusions and Recommendations}

The COV wishes to reiterate that the program mangers and science associates in the NSF/OCE Ocean Sciences Research Section are doing an excellent job. They are clearly a dedicated group who are committed to the wise use of NSF funds to support the best possible science. They are also clearly committed to making accurate, fair, and unbiased decisions based on objective data and reviews. We note that program managers have significant authority to make funding decisions, based on their overall assessment of a proposal and its importance, even if their decision is not

Table 5

Success Rates for Underrepresented Groups

\begin{tabular}{|c|c|c|c|c|}
\hline \multirow[b]{2}{*}{ Program } & \multicolumn{2}{|c|}{ African American } & \multicolumn{2}{|c|}{ Hispanic } \\
\hline & $\begin{array}{l}\text { Number of } \\
\text { Proposals }\end{array}$ & Number Awarded & $\begin{array}{c}\text { Number of } \\
\text { Proposals }\end{array}$ & Number Awarded \\
\hline
\end{tabular}

1993

$\begin{array}{lllll}\mathrm{BO} & 1 & 0 & 7 & 3 \\ \mathrm{CO} & 2 & 0 & 7 & 3 \\ \mathrm{PO} & 1 & 0 & 6 & 0 \\ \mathrm{MGG} & 2 & 0 & 7 & 3\end{array}$

\begin{tabular}{lcccc} 
& & & \\
BO & 5 & 0 & $109+$ & 2 \\
CO & 1 & 0 & 3 & 1 \\
PO & 0 & 0 & 7 & 3 \\
MGG & 1 & 0 & 6 & 0 \\
\hline
\end{tabular}


in agreement with the recommendations of letter reviews and panel reviews. We believe that this is an important and valuable responsibility and authority granted to the program managers, and we have seen no evidence in our review that this authority has been misused. It is an area that requires continued vigilance, however.

The COV made a number of specific recommendations to NSF in their final report. Following is a brief synopsis of those recommendations.

The COV was unclear as to the criteria and methodology used by OCE to assign funding to programs. Funding reallocation decisions are clearly being made, and the COV believed that a clear explanation of the financial allocation process be given to the community served by OCE because of the large impact of this process on science. Such routine disclosure will help maintain the community's confidence in OCE. particularly in these times of severe funding constraint.

The COV encouraged NSF to emphasize its policy of program managers contacting the principal investigator by telephone (or by e-mail if necessary) to inform him/her of the decisions made regarding a proposal directly after the making of these decisions (e.g., many decisions are clear after a panel, and principal investigators should be informed within a week). Written evaluations (mail reviews and panel summary) and telephone conversations should follow as soon as possible, but it is important that the program managers have sufficient time to prepare the summaries and discussions in a comprehensive and thoughtful manner.

The COV encouraged OCE to begin to collect the data necessary to evaluate the length of time between final budget revision and receipt of the awarded funds by the principal investigator's institution. and how well requested start dates are matched by dates of actual receipt of the awarded funds by the principal investigator's institution.

There was some concern expressed by the $\operatorname{COV}$ about the way review scores are recorded by NSF, because many reviewers give scores "in-between" regular categories, but only single integer scores are recorded by NSF. This can cause some confusion. We suggested that the actual scores recorded for panel and mail reviews be in a decimal format and that decimal format be used for individual average scores in the archived data base.

At the present time PIs see reviewers' comments after panel meetings and after funding decisions have been made. The COV believed it would be better if PIs could have the opportunity to respond to anonymous referees' comments before the panel meeting. The COV believes that feedback from the proposer, after receiving the mail reviews, will increase the accuracy of the funding process. While recognizing that this could add time to the overall process, the COV suggested that, on a trial basis, OCE should consider designing and operating a system to enable PIs to comment on referees' reports before their consideration by the panel.

The COV recognizes that the Large Science Programs in OCE have resulted in increased funding in oceanography and may be critical to the maintenance of the level of research activity we have enjoyed over the last decade. However, the COV believes that it is time to examine the impact of these programs on the field and on NSF's mission. Thus the COV suggested that NSF consider appointing outside review committees for the Large Science Programs to examine the balance between core program funding and Large Science Program funding in the various OSRS disciplinary programs; the administrative structure of the Large Science Program projects (e.g., steering committee composition, power, and turnover; relative influence of steering committee, panel, and program directors, etc.) and whether or not they are consistent with NSF's mission; the time line of the individual Large Science Program projects, and the feasibility of structuring a "sunset clause" for each project to ensure renewal and innovation; the effect of these programs on education, especially learning to do science in groups and the perception of science as a possible future career; the effect of these programs on collegiality within the oceanographic community; and the overall impact of these programs on our understanding of the ocean.

By 1998, the first cohorts of students from many of the REU and minority REU sites and programs will have begun to complete their graduate degrees. The COV suggested that the next COV should evaluate the success of the REU sites and minority REU sites in improving recruitment and training of students from underrepresented groups.

OCE has been actively working to increase the participation of underrepresented groups. To enhance the opportunities for people from underrepresented groups in oceanography, it is necessary to be aware of the identity of the individuals and. in some sense, to keep track of the reasons for the success or failure of the individuals in the proposal process, and the COV suggested that OCE continue and enhance its efforts to develop appropriate actions to increase the participation of underrepresented groups and persons with disabilities.

The COV encouraged OCE to continue and expand the use of non-U.S. reviewers for proposals, although there appears to be a rather low return rate from foreign reviewers. Non-U.S. reviewers should be contacted electronically (by email, fax, or telephone) to ascertain their willingness to comment on a particular proposal by the due date. Given agreement, the documentation would be sent to them by express mail (or fax) with a request for the review to be returned by e-mail or fax.

Statistical information provided to us was an important and necessary part of our review. We believe that future COVs should receive a standard pack of statistical information in a format that enables trends to be readily identified from previous COV reports. This data pack could be supplemented by additional information requested by a particular COV. 